\title{
Assessment of Unmet Obstetrics Needs in Emergency Obstetrics Services in Nekemte Referral Hospital, East Wallaga Zone, Oromiya Region, Ethiopia
}

\author{
Mitiku Getachew Kumara ${ }^{1 *}$, Hundesa Garoma ${ }^{2}$ \\ ${ }^{1}$ Department of Obstetrics and Gynecology Nekemte Hospital, Oromia
}

${ }^{2}$ Department of Obstetrics and Gynecology, Bale General Hospital, Oromia

Submission: August 18, 2019; Published: October 01, 2019

*Corresponding author: Mitiku Getachew Kumara, Department of Obstetrics and Gynecology Nekemte Hospital, Uganda

\begin{abstract}
Summary
Backgrounds: Obstetric needs are health problems that necessitate Emergency Obstetric Care services or interventions. The sum of incidence of pathologies (obstetric emergencies and complications) is taken as an indicator of obstetric need. Maternal mortality in developing countries remains high due to lack of appropriate emergency obstetric care. Major obstetric intervention (MOI) rates can be used as an indicator of unmet obstetric needs and quality of care.
\end{abstract}

Objectives: The general objective of the study was to assess unmet obstetrics need in emergency obstetric services at Nekemte referral hospital, Oromia.

Methodology: From April1 up to APRIL 30 a retrospective cross-sectional study was conducted in Nekemte referral hospital to assess the unmet obstetric needs in emergency obstetric services in the last two years (2010 and 2012) using a pretested questionnaire. Data was be analyzed using SPSS version 16.0.

Result: Out of 1015 clients took MOI during the two years period, 912 are eligible. 74(8.1\%) are less than 20 years and 529(58\%) are greater than 34 years. About three quarters (74.00\%) of the clients were from rural area. the distribution of obstetric history of the clients. 424 (46.4\%) clients are Primi paras and 95(1.2\%) are grand multi paras. 566(62.3\%) have no ANC follow up. 303(33.2\%) had obstetric problems with anemia the leading problem (7.3\%) and heart disease the least common problem (.5\%). Binary logistic regression performed to see the association between maternal and neonatal death and socio demographic factors, obstetric factors and complications. In this analysis, both the crude and adjusted analysis showed that mothers who came from rural area had 3 times increased risk of death compared to women from urban area $\left(\mathrm{AOR}=3,65(1.91,6.98) \mathrm{p}=0.001^{*}\right)$. Age $20-34$ has $18 \%$ less chance having maternal death compared to age less than 20 . (AOR=0.183(0.041, 0.823) $\mathrm{p}=0.024^{*}$ ) COR shows that mothers who had no ANC follow up had 3 times increased risk of maternal death when compared to those who had ANC follow up. (COR=3.081(1.585,5.990) $\mathrm{p}=0.001)$.Neonatal outcome was also markedly affected by place of residence. Both COR and AOR showed that neonates born form mothers who came from rural areas had 3 times increased risk of death compared to urban residence AOR $=3.6559(2.101,6.373) \mathrm{p}=0.001^{*}$ Maternal immediate complication has statistically significant association with outcome of the neonate. AOR showed that neonates born from mothers who had immediate (5days) complication had 13.2 times increased risk of death.

Keywords: Absolute; Intervention; Unmet need; Neonate

Abbreviations: AMI: Absolute Maternal Indications; ANC: Antenatal Care; BOC: Basic Obstetric Care; CBR- Crude Birth Rate; CIA: Central Intelligence Agency; CPR: Contraceptive Prevalence Rate; CSA: Central Statistics Authority; CS: Caesarean Section; DHS: Demographic Health Survey; EB: Expected Birth; EMOC: Emergency Obstetric Care; FHU: Family health Unit; HF: Health Facilities; IMR: Infant Mortality Rate; MMR: Maternal Mortality Ratio; MOH: Ministry of Health; FMOH: Federal MOH; MOI: Major Obstetric Interventions; MON: Met Obstetric Needs; MPS: Making Pregnancy Safer; OL: Obstructed Labor; TFR: Total Fertility Rate ; UON: Unmet Obstetric Need; UONN: Unmet Obstetric Need Network

\section{Background}

\section{Introduction}

Obstetric needs are health problems that necessitate EmOC services or interventions. The sum of incidence of pathologies (obstetric emergencies and complications) is taken as an indicator of obstetric need. During the 1990s the international community has made a major effort to better document the magnitude of

maternal health problems, and particularly maternal mortality. To measure the extent of this staggering human tragedy, and the failure of many health systems to meet the most basic needs for health care of the women in their constituencies, widespread use was made of maternal mortality ratios (MMR). Although this was pivotal in mobilizing policy makers it was clearly not enough 


\section{Global Journal of Reproductive Medicine}

to understand what works, what works not and what should be done. The 'First Safe Motherhood Decade' also saw a number of initiatives that tried to go beyond documentation and advocacy. Mother Care focused on improving the quality of hospital obstetric care and referral systems. The 'Prevention of Maternal Mortality Program of Columbia University and their Averting Maternal Death and Disability Initiative' promoted the notion of Emergency Obstetric Care, the use of process indicators and the reliance on networks and human rights principles. WHO marketed the concepts of Basic and Comprehensive Essential Obstetric care? The Unmet Obstetric Needs Network, the subject of this report, was part of this same movement: an attempt to go beyond the mere measurement of the extent of maternal health problems, and to find inroads to do something about it. In 1997 the European Commission and a number of other agencies decided to launch a formal Unmet Obstetric Needs Network. This UON network brought together ministries of health, development organizations, scientific institutions and practitioners who wanted to map unmet need for "major obstetric interventions for absolute maternal indications" as a starting point - to improve maternal health care and the overall functioning of their health care system. The network had to develop and test the UON approach, provide technical support as well as opportunities to learn from each other for national teams involved in this kind of work, and help countries to improve the way their health care systems deal with maternal health. The assessment of UON has never been articulated in studies conducted on EmOC in Ethiopia. Improving maternal health by reducing maternal mortality constitutes the fifth Millennium Development Goal and represents a key public health challenge in Ethiopia. In response to the need to evaluate and monitor safe motherhood interventions, this study aims at assessing gaps and deficits in MOI for AMI in comparison to the existing need.

\section{Statement of the Problem}

One of the most important factors influencing the RH status of Ethiopians is poverty. Ethiopia is one of the poorest countries in the world, with an estimated GNP per capita income of US $\$ 100$ (World Bank, 2004) [1]. Even though the poverty head count in Ethiopia was reported to have declined marginally from $45.5 \%$ in 1995 to $44.2 \%$ in 2000 , the absolute number of people living in poverty has increased steadily [1]. Today it is estimated that 47 percent of the population still lives below the poverty line $\mathrm{MOH}$, 200a: 3). Most Ethiopian women lack the reproductive and social self-determination needed to exercise their reproductive rights - a condition that, in turn, perpetuates their low RH and social status. Basic indicators clearly demonstrate the disadvantaged position of women within Ethiopian society. Women are half as likely to be literate as their male counterparts, 30 percent less likely to be employed, and marry on average, seven years earlier. Women's health is directly affected by the social and institutional context in which they live. Issues such as their low socioeconomic status, HTPs, especially FGC, early marriage, and low female literacy, all have a direct negative impact on women's health.
The MMR in Ethiopia is estimated to be $470 / 100,000$, the TFR is estimated to be 5.4 and CPR is $14 \%$. More than half of the women with age 30 years and above had their first birth in their teens. The proportion of women attending ANC is $45 \%$ with wide variation in Rural (22\%) and Urban (67\%) respectively. (DHS 2005) Trained worker assists only $25 \%$ of the deliveries. (Health Indicators MOH 2003) Obstetric Needs are health problems that necessitate EmOC services or interventions during pregnancy and labor. The concept of need integrates the epidemiological and mathematical value. The sum of incidence of pathologies (obstetric emergencies and complications) is taken as an indicator of obstetric need. The concept of unmet needs is elaborated below Number of Unmet Need $=$ Number of Problems Expected in specified population and period - Number of Problems (UN) adequately dealt with in a population and a specific period. The obstetric need is expressed in terms of health problems, which necessitate an intervention, by the health care system. Among indications for intervention some are more essential to the survival of the mother (and the child) than others. These are called "absolute maternal indications (AMI)"; and it is on this basis that the indicator of obstetric need is constructed. It is necessary, if the indicator is confined to major obstetric interventions for absolute maternal indications (MOI/AMI), to select these in such a way as to leave least room for discussion and in a way that is most relevant to the planning of the minimum interventions required. The AMI are severe ante-partum hemorrhages caused by a placenta praevia or a retro-placental hematoma (premature detachment of a normally inserted placenta), incoercible post-partum hemorrhages, major foeto-pelvic disproportions (due to a narrow pelvis or a hydrocephaly), transverse positions (shoulders neglected) and face presentations. The major obstetric interventions for absolute maternal indications comprise Caesareans, laparatomies, hysterectomies, internal versions, craniotomies or embryotomies and symphysiotomy which may be carried out to avoid a caesarean in a case of cephalo-pelvic disproportion. These interventions are selected not only because they are designed to save the mother's life (for the indications mentioned above) but also because they can be performed only in hospital. This last point is particularly important, since it enables the collection of data to be restricted to maternity institutions with operating facilities.

Process evaluation will adequately inform the design or management of maternal health care strategies if the processes of care that are being measured are those that are critical for improving maternal health so that levels and trends in maternal mortality can be inferred from their measurement. Strategies ensuring that all pregnant women have access to a health professional for delivery, for example, are thought to be critical for maternal mortality reduction, and the proportion of births with a skilled attendant has now become a widely promoted indicator for monitoring progress towards maternal mortality reductions. Similarly, ensuring access to specialized obstetric care is deemed essential for the reduction of maternal mortality, and many developing countries are now adopting the target of a 
minimum of $15 \%$ of births to take place in an essential obstetric care (EOC) facility or $5 \%$ of births with a Caesarean section [2]. Vigorous evidence linking levels of maternal mortality with specific programmed inputs is scant however, and the extent to which population-based indicators of access to obstetric services reflect levels of maternal mortality is uncertain [3].

\section{Materials and Methods}

\section{Study Area and Study Period}

Nekemte referral hospital is found in Nekemte town. Nekemte town is located in the Western part of Ethiopia, Oromia region, east wollega zone, $331 \mathrm{~km}$ far away From Addis Ababa. It is a town situated on an area of 31.92 hectares.

\section{Study Design}

Retrospective Cross-sectional study from April1, 2013 up to April 30, 2013 using quantitative and qualitative methods was used to assess Unmet Obstetric Needs in Nekemte referral hospital during the last two years (2011 \&2012) [4].

\section{Population}

Source population: All pregnant women delivered in Nekemte referral hospital during the two years period (2011\&, 2012).

Study population: All mothers who have AMI and all mothers who have received MOI in Nekemte referral Hospitals during the last two years, 2011 \& 2012were included as a sample of the study.

\section{Sample Size and Sampling Procedure}

Sample size: All patients who took MOI the year 2011 \& 2012 whose cards were available and complete were the sample size.

Sampling procedures: In this study, Nekemte referral hospital was selected to study pregnant mothers who received Major Obstetric Interventions and who have AMI during the two years period (2011 \& 2012). All patients who received MOI and all patients who have AMI during, 2011 \&2012 were involved in the study. Modified Standard questionnaire was used to collect the data.

\section{Data Collection Procedure}

The information was gathered from hospital records. These were theatre registries, delivery books/obstetric records and other relevant documents. Data collectors were midwives who have been working in the labor wards of the hospital. They were preferred because of their orientation on the technical terms stated in the formats and the fact that they read the registries better as they handle most of the records in their Units.

\section{Data Analysis Procedure}

The collected data was cleaned, coded and fed to Statistical package for social sciences (SPSS) version 16.0. The data will be cleaned again for inconsistencies and missing values. After categorizing and defining variables descriptive analyses, comparison of proportions and OR was calculated. Significances were established within $95 \%$ confidence limits. The calculation of the UON used the formula "UON = EB*RR - MOI/AMI". EB= number of expected births RR=Reference ratio (derived from maternal mortality ratios for Nekemte) $\mathrm{MOI} / \mathrm{AMI}=$ number of major obstetric interventions done for absolute maternal indication.

\section{Result}

The age distribution of the clients is presented in table one below. Out of 1015 clients took MOI during the two years period, 912 are eligible. $74(8.1 \%$ ) are less than 20 years and $529(58 \%)$ are greater than 34 years. About three quarters $(74.00 \%)$ of the clients were from rural area. Table1 distribution of socio demographic characteristics of Nekemte referral hospital, west Ethiopia, 2011 and 2012. Table2 below shows the distribution of obstetric history of the clients. 424(46.4\%) clients are Primi paras and 95(1.2\%) are grand multiparas. 566(62.3\%) have no ANC follow up. 303(33.2\%) had obstetric problems with anemia the leading problem $(7.3 \%)$ and heart disease the least common problem (.5\%). In the Table3 below, eight hundred two (87.8\%) of woman in the study had delivered at health institution; whereas $110(12.1 \%)$ had delivered at home. Among the major obstetric interventions undertaken during the two years, 737 (80.8\%) are caesarean section and $5(0.5 \%)$ are curettage. $217(23.8 \%)$ of the interventions had immediate complications with the commonest complication being hemorrhage with frequency of $88(9.6 \%)$ followed by sepsis with frequency of 66(7.2\%) [5]. The least common complication is anesthesia complication with frequency of $7(0.8 \%)$. The number of MOI done without AMI was 285 where majority were fetal distress $127(35.9 \%)$ and $163(57.2 \%)$. Other non-absolute maternal indications account for $122(42.8 \%)$ Table 4.

Table 1: Distribution of Socio Demographic Characteristics of nekemte Referral Hospital, West Ethiopia, 2011 and 2012.

\begin{tabular}{|c|c|c|}
\hline Variable & Frequency & Percent \\
\hline & Address & \\
\hline \multirow[t]{2}{*}{ urban } & 237 & \\
\hline & & 26 \\
\hline \multirow[t]{2}{*}{ Rural } & & 74 \\
\hline & 675 & \\
\hline \multirow[t]{3}{*}{ Total } & & 100 \\
\hline & 912 & \\
\hline & Age & \\
\hline \multirow[t]{2}{*}{$<20$} & & 8.1 \\
\hline & 74 & \\
\hline $20-34$ & 309 & 33.9 \\
\hline$\geq 34$ & 529 & 58 \\
\hline \multirow[t]{2}{*}{ Total } & & 100 \\
\hline & 912 & \\
\hline
\end{tabular}




\section{Global Journal of Reproductive Medicine}

Table 2: Distribution of Obstetric History of Clients of Nekemte Referral Hospital, West Ethiopia, 2011 and 2012.

\begin{tabular}{|c|c|c|}
\hline Variable & Frequency & Percent \\
\hline \multicolumn{3}{|c|}{ Parity of the Mother } \\
\hline para1 & 424 & 46.5 \\
\hline Para 2-4 & 393 & 43.1 \\
\hline$\geq$ Para5 & 95 & 10.4 \\
\hline Total & 912 & 100 \\
\hline \multicolumn{3}{|c|}{ ANC Follow up the Mother } \\
\hline yes & 346 & 37.9 \\
\hline no & 566 & 62.1 \\
\hline Total & 912 & 100 \\
\hline \multicolumn{2}{|c|}{ Present Obstetrics Problems } \\
\hline yes & 303 & 33.2 \\
\hline no & 609 & 66.8 \\
\hline
\end{tabular}

Table 3: Distribution of Place of Delivery, Mio \& Type of Immediate Complication of Nekemte Referral Hospital, West Ethiopia, 2011 and 2012.

\begin{tabular}{|c|c|c|}
\hline Variable & Frequency & Percent \\
\hline \multicolumn{3}{|c|}{ Place of delivery } \\
\hline home & 110 & 12.1 \\
\hline Institutional & 802 & 87.8 \\
\hline total & 912 & 100 \\
\hline \multicolumn{3}{|c|}{ Major obstetrics intervention } \\
\hline $\mathrm{c} / \mathrm{s}$ & 737 & 80.8 \\
\hline Hysterectomy & 39 & 4.3 \\
\hline Uterine repair & 12 & 1.3 \\
\hline Destructive delivery & 41 & 4.5 \\
\hline Blood transfusion & 51 & 5.6 \\
\hline $\begin{array}{c}\text { Manual removal of } \\
\text { placenta }\end{array}$ & 17 & 1.9 \\
\hline Curettage & 5 & 0.5 \\
\hline Other & 10 & 1.1 \\
\hline Total & 912 & 100 \\
\hline
\end{tabular}

Table 4: Distribution of Indications of Moi And Type of Immediate Complication of Nekemte Referral Hospital, West Ethiopia, 2011 and 2012.

\begin{tabular}{|c|c|c|}
\hline \multicolumn{3}{|c|}{ Indication of Major Obstetrics Complication } \\
\hline AMI & 786 & 86.2 \\
\hline Non-AMI & 612 & 13.8 \\
\hline & 912 & 100 \\
\hline \multicolumn{3}{|c|}{ Type of Immediate Complication } \\
\hline YES & 217 & 23.8 \\
\hline NO & 695 & 76.2 \\
\hline Total & 912 & 100 \\
\hline
\end{tabular}

From 912 cases during the two years period, $0.7 \%$ (6) are complicated, $0.9 \%(8)$ are referred to other health institution and 515.6\%) died. Among the causes of maternal death, hemorrhage accounts for 51\% (26) followed by pregnancy induced hypertension. 2\% (1) of causes of death are unknown. $9.3 \%(85)$ of neonates were stillborn, 5.5\% (50) were died within 24 hours and 4.4\% (40) were IUFD [6]. The most common indication for MOI is CPD with frequency of $180(19.7 \%)$ followed by malpresentation with frequency of $163(17.9 \%)$.The least common indication is abruption placenta which is $16(1.8 \%)$. Among indications, obstructed labor accounted for 131(14.4\%) from which $110(84 \%)$ are rural in residence. Uterine rupture accounted for $49(4.6)$ of the indications of which $42(85.7 \%)$ are rural in residence. PPH is more common in urban residence. Among maternal deaths, 98\% (50) occurred after intervention 2\% (1) occurred before intervention. Overall 174 (19.1\%) newborn were died Table 5.

Table 5: Distribution of Condition Mother at Discharge, Clients of Nekemte Referral Hospital, West Ethiopia, 2011 and 2012.

\begin{tabular}{|c|c|c|}
\hline Variable & Frequency & Percent \\
\hline \multicolumn{3}{|c|}{ Maternal Condition at Discharge } \\
\hline recovered & 849 & 93.1 \\
\hline Complicated and died & 63 & 6.9 \\
\hline Total & 912 & 100 \\
\hline \multicolumn{2}{|c|}{ Outcome of New Borne } \\
\hline Alive & 738 & 80.9 \\
\hline Died & 174 & 19.1 \\
\hline
\end{tabular}

\section{Quantification of UON}

The UON is based on the following concept:

Number of Unmet Need $=$ Number of Problems Expected Number of Problems (UN) in a population adequately dealt with, the calculation of the UON used the formula "UON = EB*RR- MOI/ AMI" EB*RR represents the MOI/AMI expected

1. $\mathrm{EB}=69000$

2. In the general circumstances $2 \%$ of births get complicated or require MOI. The EB that needed MOI therefore becomes 2760

3. $15 \%$ of births need MOI.

4. MOI/AMI= 627 (Major Obstetric Intervention actually performed for Absolute Maternal Indication in the same population in the same period).

The UON is therefore $2760 * 0.15-311=2133.2133$ births which needed major obstetric intervention in Addis Ababa during the two years period did not receive it. In other words, the deficit of MOI/AMI per 100 births is 9.46 . Table 6 shows that the actual number of MOI for AMI is below the minimum benchmark of $2 \%$. The overall level of MOIs for AMIs per EB during the study period was $77 \%$. Using the benchmark of $2 \%$, the absolute deficit was 2133 interventions (or 1066 per year) Figure 1. 
Table 6: Expected number of MOI for AMI VS actual number performed.

\begin{tabular}{|c|c|c|c|c|}
\hline $\begin{array}{c}\text { Expected Births (EB) per } \\
\mathbf{2} \text { years }\end{array}$ & $\begin{array}{c}\text { Need for MOIs for AMIs } \\
\text { per year (2\%) }\end{array}$ & $\begin{array}{c}\text { Actual Number of MOI for } \\
\text { AMI * }\end{array}$ & Deficit in MOIs & MOI for AMI for EB as \% \\
\hline 138000 & 2760 & 627 & 2133 & $77 \%$ \\
\hline
\end{tabular}

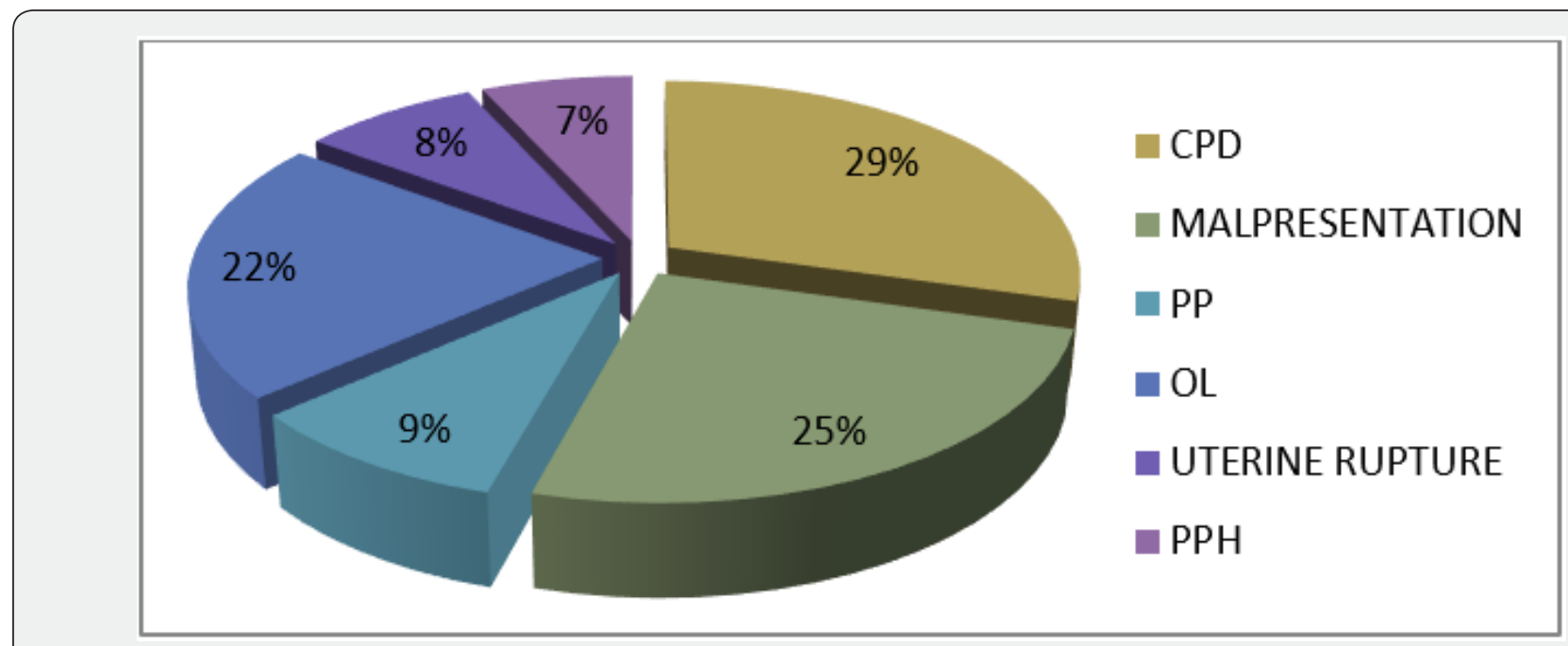

Figure 1: Distribution of Outcome of The Mother \& the Neonate of Nekemte Referral Hospital, West Ethiopia, 2011 and 2012.

Factors Associated with Maternal and Neonatal Outcomes

Binary logistic regression performed to see the association between maternal and neonatal death and sociodemographic factors, obstetric factors and complications. In this analysis, both the crude and adjusted analysis showed that mothers who came from rural area had 3 times increased risk of death compared to women from urban area $\left(. \mathrm{AOR}=3,65(1.91,6.98) \mathrm{p}=0.001^{*}\right)$. Age 20-34 has $18 \%$ less chance having maternal death compared to age less than 20. $\left(\mathrm{AOR}=0.183(0.041,0.823) \mathrm{p}=0.024^{*}\right)$. COR shows that mothers who had no ANC follow up had 3 times increased risk of maternal death when compared to those who had ANC follow up. (COR=3.081(1.585,5.990) p=0.001). Figure 2 Neonatal outcome was also markedly affected by place of residence. Both COR and AOR showed that neonates born form mothers who came from rural areas had 3 times increased risk of death compared to urban residence. AOR $=3.6559(2.101,6.373) \mathrm{p}=0.001$ * Maternal immediate complication has statistically significant association with outcome of the neonate. AOR showed that neonates born from mothers who had immediate (5 days) complication had 13.2 times increased risk of death Table 7.

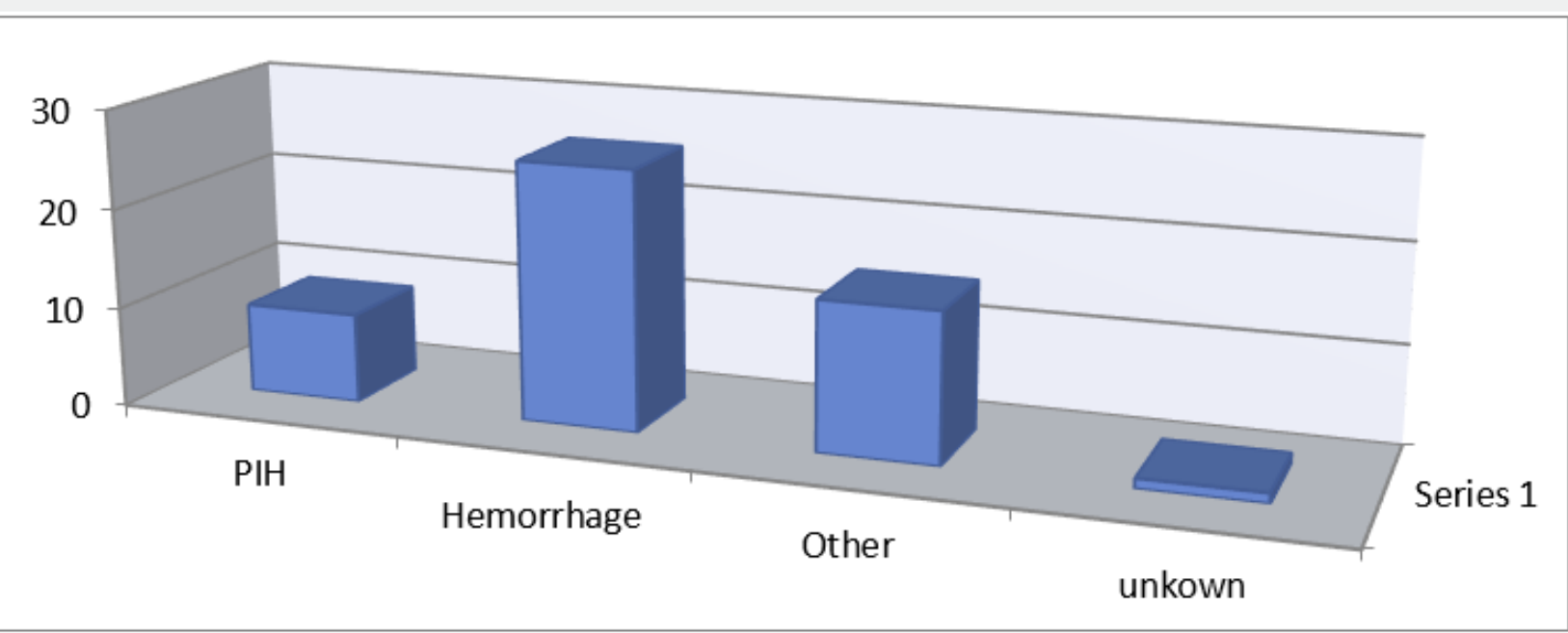

Figure 2: Causes of Maternal Death of Nekemte Referral Hospital, West Ethiopia, 2011 and 2012. 
Table 7: Binary logistic regression for factors associated with maternal mortality.

\begin{tabular}{|c|c|c|c|c|c|}
\hline \multirow{2}{*}{ Variable } & \multirow{2}{*}{ Category } & \multicolumn{2}{|c|}{ Condition of mother at discharge } & \multirow{2}{*}{ COR } & \multirow{2}{*}{ AOR } \\
\hline & & Recovered & Complicated and died & & \\
\hline \multirow{2}{*}{ Address } & Urban & 231 & 6 & 1 & 1 \\
\hline & Rural & 618 & 57 & $3.551(1.511,8.347) \mathrm{p}=0.004^{*}$ & $3,65(1.91,6.98) \mathrm{p}=0.001^{*}$ \\
\hline \multirow{3}{*}{$\begin{array}{l}\text { Age of the } \\
\text { mother }\end{array}$} & $<20$ & 59 & 15 & 1 & 1 \\
\hline & $20-34$ & 298 & 11 & $.387(.230, .650) \mathrm{P}=.0001^{*}$ & $0.183(0.041,0.823) \mathrm{p}=0.024^{*}$ \\
\hline & $\geq 34$ & 492 & 17 & $.680(.412,1.123) \mathrm{P}=.132$ & $0.960(0.533,1.73) p=0.892$ \\
\hline \multirow{2}{*}{ ANC } & No & 514 & 52 & $3.081(1.585,5.990) * \mathrm{p}=0.001$ & $1.144(.691,1.894) \quad$ p.601= \\
\hline & Yes & 335 & 11 & 1 & 1 \\
\hline \multirow{2}{*}{ Place of delivery } & Institution & 755 & 47 & 1 & 1 \\
\hline & Home & 94 & 16 & $3.081(1,585,5.990) \mathrm{P}=0.001$ & $1.65(0.876,3.13)$ \\
\hline \multirow{2}{*}{$\begin{array}{l}\text { Immediate } \\
\text { complication }\end{array}$} & No & 692 & 3 & 1 & 1 \\
\hline & Yes & 157 & 60 & $11.714(8.046,17.053) \mathrm{P}=.0001^{*}$ & $8.989(5.52,14.6) \mathrm{p}=0.001^{*}$ \\
\hline
\end{tabular}

\section{Discussion}

Eight hundred two (87.8\%) of woman in the study had delivered at health institution; whereas $110(12.1 \%)$ had delivered at home. Among the major obstetric interventions undertaken during the two years, $737(80.8 \%)$ are caesarean section and $5(0.5 \%)$ are curettage. $217(23.8 \%)$ of the interventions had immediate complications with the commonest complication being hemorrhage with frequency of $88(9.6 \%)$ followed by sepsis with frequency of $66(7.2 \%)$. The least common complication is anesthesia complication with frequency of $7(0.8 \%)$. The number of MOI done without AMI was 285 where majority were fetal distress $127(35.9 \%)$ and 163(57.2\%). Other non-absolute maternal indications account for $122(42.8 \%)$ it was high incase institutional delivery and low with home delivery possible reason is expansion of health institution, distribution of skilled manpower and arrangement of referral system. [7,8]. The most common indication for MOI is CPD with frequency of $180(19.7 \%)$ followed by malpresentation with frequency of $163(17.9 \%)$.The least common indication is abruption placenta which is $16(1.8 \%)$. Among indications, obstructed labor accounted for 131(14.4\%) from which $110(84 \%)$ are rural in residence. Uterine rupture accounted for $49(4.6)$ of the indications of which $42(85.7 \%)$ are rural in-residence Table 8 . PPH is more common in urban residence. (3. 8) [8-11].

Table 8: Binary Logistic Regression for Factors Associated with Outcome of the Newborn.

\begin{tabular}{|c|c|c|c|c|c|}
\hline \multirow{2}{*}{ Variable } & \multirow{2}{*}{ Category } & \multicolumn{2}{|c|}{ Outcome of the Newborn } & \multirow{2}{*}{ COR } & \multirow{2}{*}{ AOR } \\
\hline & & Alive & Died & & \\
\hline \multirow{2}{*}{ Address } & Rural & 523 & 152 & 1 & 1 \\
\hline & Urban & 215 & 22 & $2.840(1.767,4.565) \mathrm{p}=0.001^{*}$ & $3.6559(2.101,6.373) p=0.001 *$ \\
\hline \multirow{4}{*}{ Parity } & Para1 & 365 & 59 & & 1 \\
\hline & & & & 1 & \\
\hline & Para2-4 & 306 & 87 & $0.283(0.080,0.996) \mathrm{P}=0.049^{*}$ & $1.738(0.80523 .629) \mathrm{p}=0.141$ \\
\hline & $\geq$ Para5 & 67 & 28 & $0.498(0.142,1.739) \mathrm{P}=0.274$ & $3.046(0.735,12.62) \mathrm{p}=0.125$ \\
\hline \multirow{2}{*}{ Immediate complication } & Yes & 103 & 114 & $11.714(8.046,17.053) \mathrm{P}=0.001^{*}$ & $13 . .219(8.687,20.116) \mathrm{p}=0.001^{*}$ \\
\hline & No & 635 & 60 & 1 & 1 \\
\hline
\end{tabular}

\section{References}

1. Mamady Cham, Johanne Sundby, SiriVangen (2009) Availability and quality of emergency obstetric care in Gambia's main referral hospital. Women users' testimonies Reproductive Health. Reproductive health journal 6(5):1-3.

2. Helen Prytherch, Siriel Massawe, Rainer Kuelker, Claudia Hunger, Ferdinand Mtatifikolo, et al. (2007) The unmet need for Emergency Obstetric Care in Tanga Region, Tanzania. BMC Pregnancy and Childbirth 7(16): 11-12.
3. C Hunger, R Kulker, H Kitundu, S Massawe, A Jahn (2007) Assessing unmet obstetric need in Mtwara Region, Tanzania. Trop Med Int Health 12(10): 1239-1247.

4. Alexander Uamai. Review of WHO handbook (2010) Monitoring emergency obstetric care, Lagos Island Maternity Hospital, Lagos, Nigeria: Geneva Foundation for Medical Education and Research.

5. Marge Koblinsky, Frances Tain, Solomon Tesfaye (2010) Reducing maternal mortality and increasing use of skilled birth attendance: Ethiopia and MDG 5. Ethiopian Journal of Reproductive Health 4(1): 4-15. 
6. WHO, UNICEF, UNFPA, and the World Bank? Maternal Mortality Estimates in 2005, WHO Press, World Health Organization, 20 Avenue Appia, 1211 Geneva 27: 1-10.

7. Usaid, fistula cre, engender health, intra health (2010) Overview of safe motherhood and global maternal morbidity and mortality, FMOH Safe Motherhood Programme, Ethiopia 1-20.

8. Dominique Dubourg, Vincent De Brouwere, Wim Van Lerberghe Fabienne Richard, Vincent Litt, et al. (2000) The Unmet Obstetric
NeedsNetwork Final, Institute of Tropical Medicine (ITM) Department of Public Health, Antwerpen / Belgium: 1-25.

9. http://www.amdd.hs.columbia.edu

10. C Ronsmans, JF Etard, G Walraven, L Høj, A Dumont, et al. (2003) Maternal mortality and access to obstetric services in West Africa. Tropical Medicine and International Health 8(10): 940-948.

11. http://www.uonn.org
This work is licensed under Creative Commons Attribution 4.0 License DOI: 10.19080/GJORM.2019.07.555704 